\title{
Strict DNA Valence Control in Ultrasmall Thiolate- Protected Near-Infrared-Emitting Gold Nanoparticles
}

Zhiyi Dai, Yue Tan, Kui He, Huarui Chen and Jinbin Liu*

Key Laboratory of Functional Molecular Engineering of Guangdong Province, School of Chemistry and Chemical Engineering, South China University of Technology, Guangzhou 510640, China

Supporting Information 


\section{Table of content}

Experimental Procedures ...................................................................................................S3

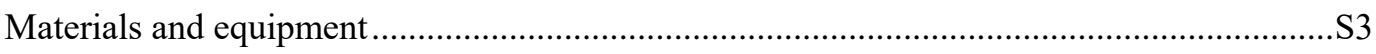

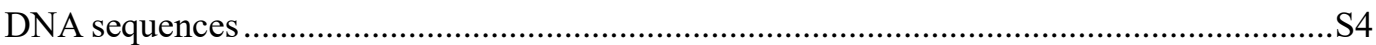

Synthesis of psDNA functionalized gold nanoparticles (psDNA-AuNPs) .............................S5

Agarose gel electrophoresis (AGE) and native polyacrylamide gel electrophoresis (PAGE).S5

Quantification of the gold contents in the gel bands................................................................. 5

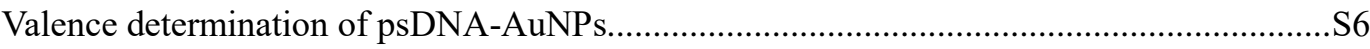

DNA decoration of glutathione-coated gold nanoparticles (GS-AuNPs) through ligand

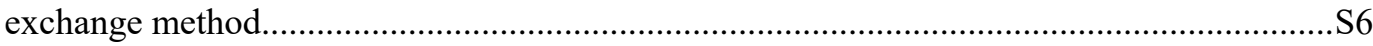

DNA decoration of GS-AuNPs through one-pot synthesis method by using 5'thiol-capped

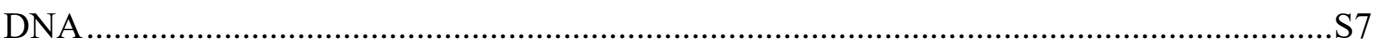

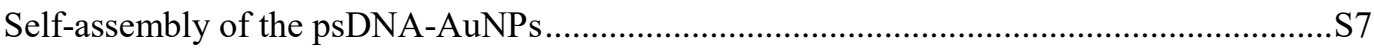

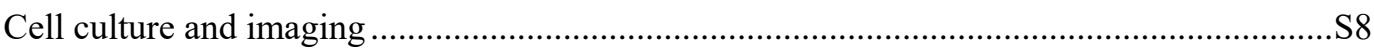

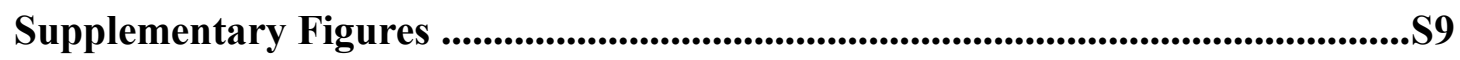

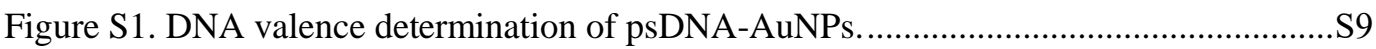

Figure S2. Characterization of psDNA stability and the related status on surface of psDNAAuNPs using $12 \%$ native PAGE.

Figure S3. Characterization of the non-canonical structure or non-specific binding of psDNA-

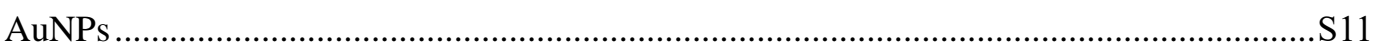

Figure S4. Typical TEM images of V1-psDNA-AuNPs ........................................................ 12

Figure S5. Size distributions of V1-psDNA-AuNPs synthesized using psDNA with different

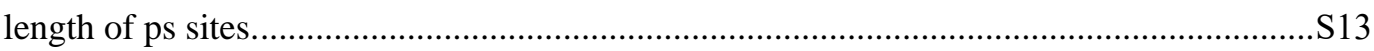

Figure S6. Typical TEM images of the GS-AuNPs fractions synthesized using psDNA with different length of ps sites.

Figure S7. Size distributions of GS-AuNPs fractions synthesized using psDNA with different length of ps sites.....

Figure S8. Determination of thiol-DNA modification of GS-AuNPs through one-pot synthesis using 5'thiol-capped DNA.

Figure S9. AGE characterization of the GS-AuNPs before and after ligand exchange with

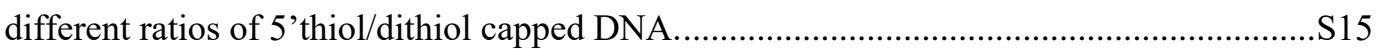

Figure S10. Schematic diagram of one-dimensional self-assemblies of psDNA-AuNPs.....S16

Figure S11. Fluorescence statistics of the corresponding cells..........................................S17

Figure S12. UV-Vis characterization of free psDNA of the psDNA-AuNPs.......................S17

Figure S13. Characterization of the purification of 20S-psDNA-AuNPs............................S18

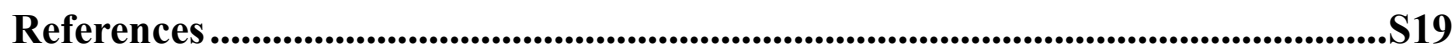




\section{Experimental Procedures}

\section{Materials and equipment}

Gold chloride trihydrate $\left(\mathrm{HAuCl}_{4} \cdot 3 \mathrm{H}_{2} \mathrm{O}\right)$ and glutathione $(\mathrm{GSH})$ were purchased from Sigma-Aldrich. The phosphorothioates (ps)-modified DNA (psDNA) and regular DNA were purchased from Sangon (Shanghai, China) and Takara (Dalian, China), which was used as received. Fetal bovine serum (FBS) was purchased from Lonsa Science Srl (USA). Phosphate buffer and tris-borate-EDTA solutions were prepared according to the standard protocols and calibrated with SevenCompact ${ }^{\mathrm{TM}} \mathrm{pH} / \mathrm{Ion}$ Meter S220-Micro (METTLER TOLEDO, Switzerland). Other chemicals were obtained from SigmaAldrich and used directly. All glassware was cleaned with fresh aqua regia before use. Ultrapure water was achieved from a Pall water purification system (Cascada III.I, USA). The luminescence spectra were collected using a LS-55 luminescence spectrophotometer (PerkinElmer, USA). The absorption spectra were obtained from a UV-Vis spectrophotometer (UV2600 Shimadzu, Japan). High resolution transmission electron microscopy (HRTEM) images were obtained with JEOL JEM 2100F TEM (Japan) with an accelerating voltage of $200 \mathrm{kV}$. The agarose gel imaging was collected with UVP ChemStudio PLUS 815 imaging system (Analytikjena, USA). 
DNA sequences

\begin{tabular}{|l|l|}
\hline nS-psDNA & $\left(\right.$ A $^{*}$ AAAAACAGTCAGGCAGTCAGTCA \\
\hline Chimeric-7S-psDNA & $\begin{array}{l}\text { CTCAGGTCACAGAATCGTAAAAAA*A*A*A*A*A* } \\
\text { A*AAAAACAGTCAGGCAGTCAGTCA }\end{array}$ \\
\hline sgc8c-aptamer & $\begin{array}{l}\text { ATCTAACTGCTGCGCCGCCGGGAAAATACTGTACGG } \\
\text { TTAGAGTGACTGACTGCCTGACTG }\end{array}$ \\
\hline cDNA & $\begin{array}{l}\text { AACAAGCCACATAGAAGAGAACAAGCCACATAGAT } \\
\text { CTGACTGACTGCCTGACTG }\end{array}$ \\
\hline cDNA1 & $\begin{array}{l}\text { AACAAGCCACATAGAAGAGAACAAGCCACATAGAT } \\
\text { CTGACTGACTGCCTGACTG }\end{array}$ \\
\hline cDNA2 & $\begin{array}{l}\text { ACGATTCTGTGACCTGAGAACAAGCCTTATAGTTG } \\
\text { AGAACAAGCCACATAGATC }\end{array}$ \\
\hline $2 \mathrm{a}$ & $\begin{array}{l}\text { TGACTGACTGCCTGACTGTGACTGACTGCCTGACT } \\
\text { G }\end{array}$ \\
\hline $3 \mathrm{a}$ & $\begin{array}{l}\text { TGACTGACTGCCTGACTGTGACTGACTGCCTGACT } \\
\text { GTGACTGACTGCCTGACTG }\end{array}$ \\
\hline $6 \mathrm{a}$ & $\begin{array}{l}\text { TGACTGACTGCCTGACTGTGACTGACTGCCTGACT } \\
\text { GTGACTGACTGCCTGACTGTGACTGACTGCCTGAC } \\
\text { TGTGACTGACTGCCTGACTGTGACTGACTGCCTGA } \\
\text { CTG }\end{array}$ \\
\hline $2 \mathrm{a}-\mathrm{M}$ & $\begin{array}{l}\text { GAACAAGCCACATAGATATGACTGACTGCCTGAC } \\
\text { TG }\end{array}$ \\
\hline $3 \mathrm{a}-\mathrm{M}$ & $\begin{array}{l}\text { AACAAGCCACATAGAAGAGAACAAGCCACATAGAT } \\
\text { CTGACTGACTGCCTGACTG }\end{array}$ \\
\hline $6 \mathrm{a}-\mathrm{M}$ & $\begin{array}{l}\text { AACAAGCCACATAGAAGAGAACAAGCCACATAGAT } \\
\text { CAACAAGCCACATAGAAGAGAACAAGCCACATAGA } \\
\text { TCAACAAGCCACATAGAAGATGACTGACTGCCTG } \\
\text { ACTG }\end{array}$ \\
\hline AAAAAAAAAAAACAGTCAGGCAGTCAGTCA \\
\hline mDNA & $\begin{array}{l}\text { AACAAGCCACATAGAAGAGAACAAGCCACATAGAT } \\
\text { CCGCTCGCTCGCTTGGAGC }\end{array}$ \\
\hline
\end{tabular}

A* means the phosophodiester backbone of a deoxynucleotide A (Adenine) is modified with phosphorothioates. 


\section{Synthesis of psDNA functionalized gold nanoparticles (psDNA-AuNPs)}

The psDNA-AuNPs were synthesized using both psDNA and GSH as reductants.

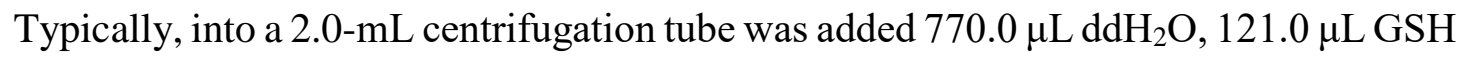
$(24.0 \mathrm{mM})$ and $20.0 \mu \mathrm{L} \mathrm{HAuCl}_{4}(145 \mathrm{mM})$ under vigorous stirring in an oil bath at $95^{\circ} \mathrm{C}$. After $2 \mathrm{~min}$, the solution became colorless, indicating the formation of GS-Au(I) complexes. The $\mathrm{pH}$ of the solution was then immediately adjusted to 8-9 with $18.0 \mu \mathrm{L}$ $\mathrm{NaOH}(1.0 \mathrm{M})$ followed by adding $148.0 \mu \mathrm{L}$ psDNA $(0.654 \mathrm{mM}$, quantified by UV absorption of $260 \mathrm{~nm}$, dissolved in $1 \times$ TE buffer, the molar ratio of GSH to psDNA was 30:1). The reaction was kept stirring at $95{ }^{\circ} \mathrm{C}$ for additional more than $2.5 \mathrm{~h}$ to achieve the desired luminescence properties. The reaction was stopped by cooling the centrifugation tube at room temperature under stirring. The resulted solution was transferred to an ultra-4 centrifugal filter device (Amicon, $30 \mathrm{k}$ MWCO) spinning at $15000 \mathrm{~g}$ for $5 \mathrm{~min}$ to remove the excess GSH. The formed psDNA-AuNPs was then precipitated with $35 \%$ ethanol and $0.5 \mathrm{M} \mathrm{NaCl}$ and $20 \mathrm{mM} \mathrm{MgCl}_{2}$ solution to remove the excess psDNA in the supernatant (Figure S12). The psDNA-AuNPs precipitates were then dissolved in $1 \times$ TE buffer for further use. Besides, a fast ethanol precipitation method was developed to remove byproduct GS-AuNPs from 20S-psDNA-AuNPs (Figure S13). Reactions for different ratio values of GSH to psDNA (e.g., 30:1, 100:1 and $300: 1$ ) or ps base length of psDNA (1S to 20S) were conducted similarly. The GSHcoated gold nanoparticles (GS-AuNPs) without adding psDNA were also synthesized and used as control.

\section{Agarose gel electrophoresis (AGE) and native polyacrylamide gel electrophoresis} (PAGE)

The samples were diluted into $0.5 \times$ TE solution ( $\mathrm{pH} 8.0$ ) with $7.5 \%$ glycerol, $500 \mathrm{mM}$ $\mathrm{NaCl}$ and $20 \mathrm{mM} \mathrm{MgCl}_{2}$ to a final volume of $20 \mu \mathrm{L}$. The resulting solution was analyzed by $5 \%$ AGE or $12 \%$ native PAGE using a Gel Electrophoresis System (DYY-8C, China, $6 \mathrm{~V} / \mathrm{cm}, 20 \mathrm{~mA}$ ) for $60-100 \mathrm{~min}$ in $0.5 \times$ TBE buffer. All the samples migrated from negative electrode towards the positive electrode. The PAGE gels were stained by Stains-All.

\section{Quantification of the gold contents in the gel bands.}

The gold contents of psDNA-AuNPs synthesized by psDNA with different length of ps sites was analyzed by inductively coupled plasma mass spectrometry (ICP-MS) after 
separation with gel electrophoresis. Each band of the product was collected and the gold content was measured with an inductively coupled plasma mass spectrometry. Typically, the psDNA-AuNPs were separated into different bands by 5\% AGE, and these bands were imaged by imaging system, and then finally sliced carefully and collected into 20.0-mL glass bottles respectively. After addition of $4.0 \mathrm{~mL}$ aqua regia for $24 \mathrm{~h}$ digestion, these glass bottles were heated to $130{ }^{\circ} \mathrm{C}$ to vaporize all the liquid. The remaining residues were redissolved in $3 \% \mathrm{HCl}$ solution and sonicated for $1 \mathrm{~h}$. The obtained solution was filtered and diluted for following ICP-MS measurement. The gold contents of by-product band GS-AuNPs were then used as references to quantitatively evaluate the effects of ps length by analyzing the ratios of V1-psDNAAuNPs to GS-AuNP (RV1/GS) and V2-psDNA-AuNPs to GS-AuNPs (RV2/GS), respectively. The psDNA-AuNPs synthesized using Divalent-7S-psDNA as template was analyzed with a similar method as described above.

\section{Valence determination of psDNA-AuNPs}

The concentration of psDNA on the psDNA-AuNPs was determined by using tris(2carboxyethyl) phosphine (TCEP) to completely etch the AuNPs, ${ }^{1}$ and measuring the UV absorbance at $260 \mathrm{~nm}$. Typically, the AuNPs $(2-10 \mu \mathrm{L})$ was incubated with $1 \mu \mathrm{L}$ $0.1 \mathrm{M}$ TCEP $(1 \times$ TE buffer, $\mathrm{pH} 8.0)$ at $37^{\circ} \mathrm{C}$ for $2 \mathrm{~h}$. Then the corresponding amount of cDNA were added according to the ratio of psDNA to cDNA. The psDNA-AuNPs purified by ultrafiltration or gel slice was first incubated with different amount of cDNA at $37{ }^{\circ} \mathrm{C}$ for $1.5 \mathrm{~h}$ under the condition of $0.50 \mathrm{M} \mathrm{NaCl}$ and $20.0 \mathrm{mM} \mathrm{MgCl}_{2}$, and then analyzed by $5 \%$ AGE or $12 \%$ native PAGE for $60-100 \mathrm{~min}$. The bands were imaged through an imaging system using different emissive filters.

\section{DNA decoration of glutathione-coated gold nanoparticles (GS-AuNPs) through}

\section{ligand exchange method}

The GS-AuNPs was synthesized according to our previous reports with slight modifications. $^{2}$ Briefly, into a 100-mL three-necked bottle was added $3.75 \mathrm{~mL} 24.0$

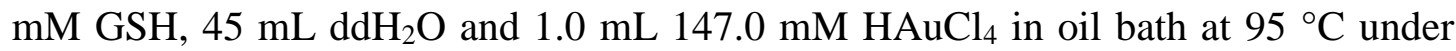
vigorous stirring. This reaction would continue for $30 \mathrm{~min}$ and stopped by cooling down to room temperature under vigorous stirring. The synthesized GS-AuNPs were adjusted to $\mathrm{pH} \sim 8.0$ purified by centrifuging at $4000 \mathrm{~g}$ for $15 \mathrm{~min}$ to remove the large aggregates. The supernatant was further purified using ethanol solution (ethanol-to- $\mathrm{H}_{2} \mathrm{O}=1: 2(v / v)$ with $\mathrm{pH}$ adjusted to $\sim 4$, followed by centrifuging at $4000 \mathrm{~g}$ for $10 \mathrm{~min}$ to discard the 
supernatant. The precipitates were then resuspended by adjusting the solution $\mathrm{pH}$ to 7 $\sim 8$, and then purified again with an ultra-4 centrifugal filter device (Amicon, $10 \mathrm{~K}$ MWCO) spinning at $15000 \mathrm{~g}$ for $5 \mathrm{~min}$ for 3 times. The purified GS-AuNPs were quantitated by UV absorption at $350 \mathrm{~nm}$ to obtain their molar concentration. ${ }^{2-3}$ The 5 'thiol/dithiol-capped DNA powder was dissolve in $1 \times$ TE buffer and immediately used after UV quantification in following procedure. The stocking solution of 5'thiol/dithiol-capped DNA after a long-term storage was treated with $\mathrm{NaBH}_{4}$ and centrifuged with Ultra-4 centrifugal filter devices (Amicon, $3 \mathrm{~K} \mathrm{MWCO}$ ) at $15000 \mathrm{~g}$ for $5 \mathrm{~min}$ to remove unreacted $\mathrm{NaBH}_{4}$ before use. The mixture of $6.4 \mu \mathrm{M}$ GS-AuNPs and $25.6 \mu \mathrm{M} 5$ 'thiol/dithiol-capped DNA was incubated in $1 \times \mathrm{PB}(\mathrm{pH} 6$, containing $0.50 \mathrm{M} \mathrm{NaCl})$ at $37{ }^{\circ} \mathrm{C}$ under shaking overnight. After overnight incubation, the solution was centrifuged with ultra-4 centrifugal filter devices (Amicon, $50 \mathrm{k} \mathrm{MWCO}$ ) to remove excess the DNA.

\section{DNA decoration of GS-AuNPs through one-pot synthesis method by using}

\section{5'thiol-capped DNA}

The whole procedure was similar to the synthesis of psDNA-AuNPs. Typically, into a 2.0-mL centrifugation tube was added $770.0 \mu \mathrm{L}$ ddH $_{2} \mathrm{O}, 121.0 \mu \mathrm{L} \mathrm{GSH}(24.0 \mathrm{mM})$ and $20.0 \mu \mathrm{L} \mathrm{HAuCl}_{4}(145 \mathrm{mM})$ under vigorous stirring in an oil bath at $95{ }^{\circ} \mathrm{C}$. After $2 \mathrm{~min}$, the solution became colorless, indicating the formation of $\mathrm{Au}(\mathrm{I})-\mathrm{GS}$ complexes. The $\mathrm{pH}$ of the solution was then immediately adjusted to 8-9 with $18.0 \mu \mathrm{L} \mathrm{NaOH}(1.0 \mathrm{M})$ followed by adding $150.0 \mu \mathrm{L}$ 5'thiol-capped DNA $(0.2 \mathrm{mM}$, quantified by UV absorption of $260 \mathrm{~nm}$, dissolved in $1 \times$ TE buffer). The reaction was kept stirring at $95{ }^{\circ} \mathrm{C}$ for additional $2.5 \mathrm{~h}$ to achieve the desired luminescence properties. The reaction was stopped by cooling the centrifugation tube at room temperature under stirring. The resulted solution was transferred to an ultra-4 centrifugal filter device (Amicon, 30k MWCO) to remove the excess GSH and DNA.

\section{Self-assembly of the psDNA-AuNPs}

The purified V1-psDNA-AuNPs and the DNA sequences with 2, 3 or 6 repeated complementary binding domains (Aptamer, 2a, 3a or 6a) were incubated in the solution containing $500 \mathrm{mM} \mathrm{NaCl}$ and $40 \mathrm{mM} \mathrm{MgCl} 2$ at $37{ }^{\circ} \mathrm{C}$ for $1.5 \mathrm{~h}$, respectively, and then cooled to room temperature. Samples incubated with different ratios of psDNA to complementary sequence were carried out with 5\% AGE in $0.5 \times$ TBE buffer for 60 - 
$100 \mathrm{~min}$. The completely hybridized assemblies were selected for TEM observations. The TEM grids were pretreated with glow discharge before use.

\section{Cell culture and imaging}

The A549 cells were cultured in Dulbecco's modified Eagle's medium (DMEM, Hyclone) supplemented with $10 \%$ fetal bovine serum (FBS) and $1 \%$ penicillinstreptomycin. The CCRF-CEM cells were cultured in RPMI 1640 medium supplemented with $10 \%$ fetal bovine serum (FBS) and 1\% penicillin-streptomycin All cells were maintained in $5 \% \mathrm{CO}_{2}$ at $37{ }^{\circ} \mathrm{C}$ and a humidified atmosphere. Cells were washed by DPBS before incubation with AuNPs. The cells incubated with the AptAuNPs or psDNA-AuNPs at $4{ }^{\circ} \mathrm{C}$ for $1 \mathrm{~h}$ in binding buffer $(1 \times$ DPBS with calcium and magnesium, containing $4.5 \mathrm{~g} / \mathrm{L}$ glucose and $5 \mathrm{mM} \mathrm{MgCl}_{2}$ ), and then washed with binding buffer before imaging. The images were taken by a fully-motorized inverted microscope system (Olympus IX83-DSU, Japan, a spinning disk confocal technology) assembled with an EMCCD (Evolve 512 Delta) through 100× oil-immersion objective under Xe-lamp excitation (Ex: 355-375 nm, Em: 765-855 nm, exposure time: $500 \mathrm{~ms}$ ). 


\section{Supplementary Figures}
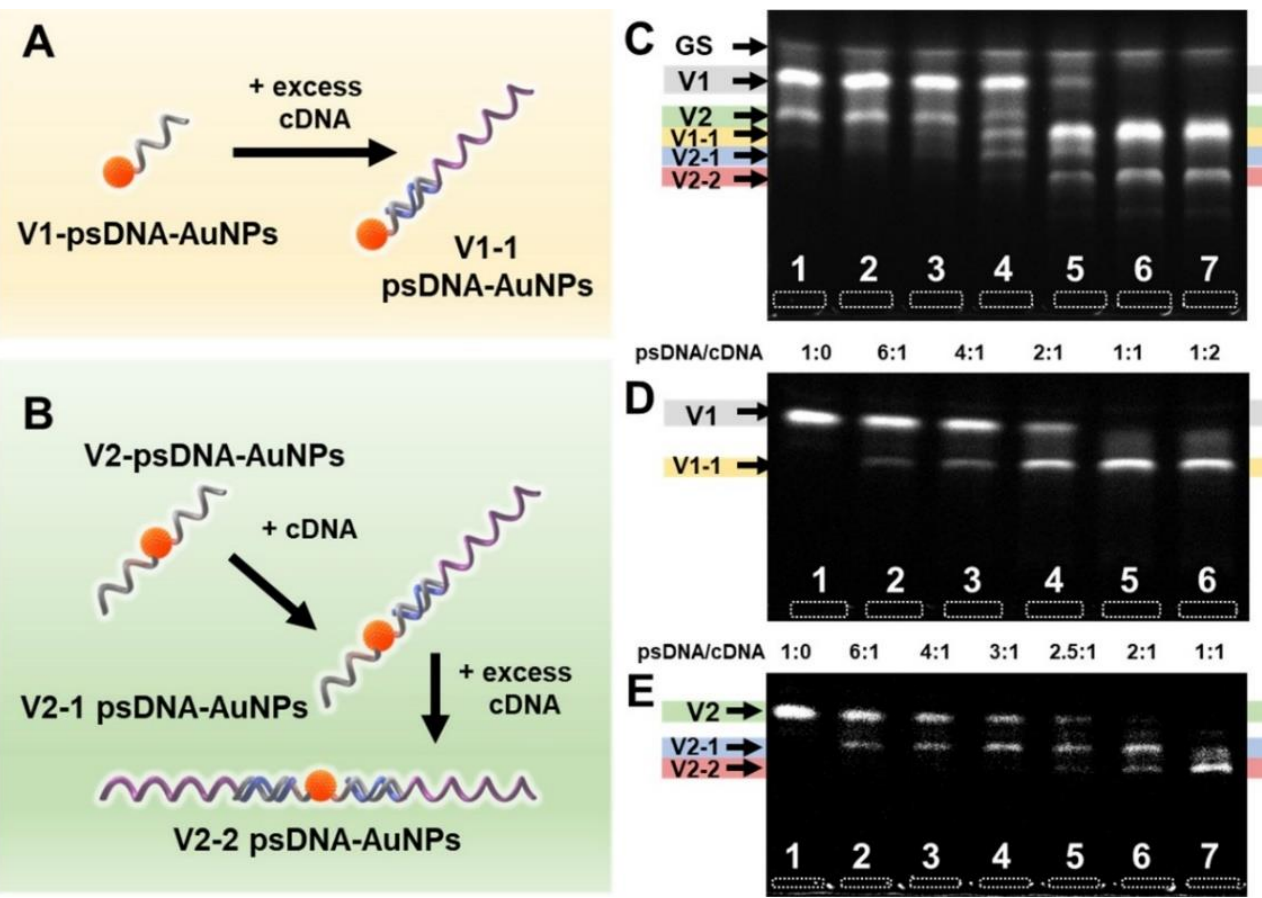

Figure S1. DNA valence determination of psDNA-AuNPs. Schematic diagram of DNA valence determination of V1-psDNA-AuNPs (A) and V2-psDNA-AuNPs (B) with one or two psDNA. (C) The AGE of unpurified psDNA-AuNPs with increase of cDNA. The AGE of purified V1-psDNA-AuNPs (D) and V2-psDNA-AuNPs (E) with increase of stoichiometric ratios of V1-psDNA-AuNPs to cDNA. The DNA valence of psDNA-AuNPs was measured with a previously reported titration strategy ${ }^{4,5}$ and observed with gel electrophoresis ${ }^{6,7}$. The binding of cDNA to psDNA-AuNPs would result in DNA valence-dependent bands during AGE. The as-formed GS-AuNPs without psDNA, exhibited low resistance with a fast migration band (GS) unresponsive to the added cDNA. All the cDNA-responsive bands (V1 and V2) were cut and recollected for individual titration. With addition of cDNA, the band V1 decreased gradually and formed only one new band (V1-1), indicating that the psDNA-AuNPs in V1 band (V1-psDNA-AuNPs) was decorated with one psDNA. As for band V2, it gradually changed to a lower migration band (V2-1) at low cDNA concentrations, but eventually converted to another much lower migration band (V2-2), demonstrating that psDNA-AuNPs in V2 band (V2-psDNA-AuNPs) had two psDNA. 


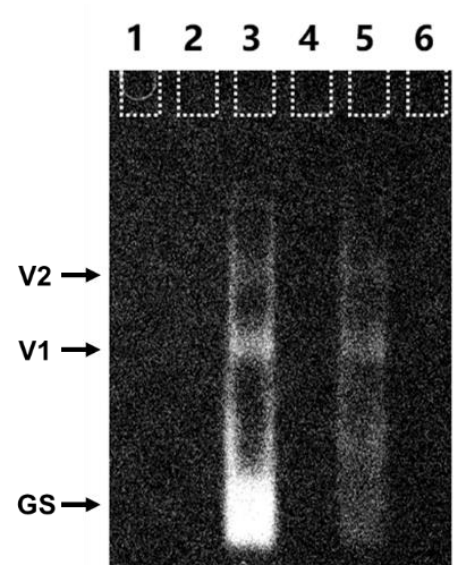

A

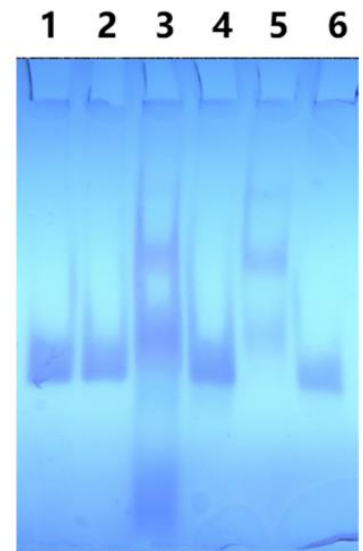

B

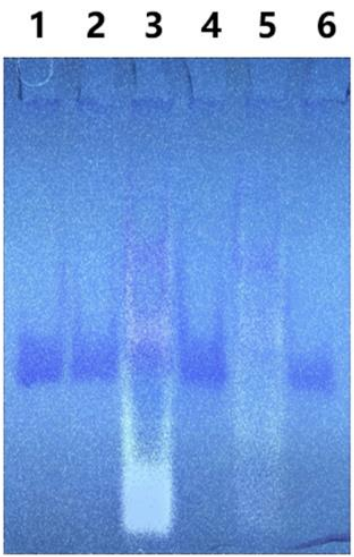

C

Figure S2. Characterization of psDNA stability and the related status on surface of psDNA-AuNPs using 12\% native PAGE. (A) Fluorescence image; (B) Bright field image; (C) Overlay of fluorescence and bright field images. Lane 1: psDNA without treatment; lane 2: psDNA under $95^{\circ} \mathrm{C}$ and vigorous stirring; lane 3: psDNA-AuNPs after ultrafiltration (10 K MWCO); lane 4: psDNA-AuNPs (similar as lane 3) treated by TCEP; lane 5: psDNA-AuNPs after ethanol precipitation); lane 6: psDNA-AuNPs (similar as lane 5) treated by TCEP. We firstly tested the stability of the psDNA under $95^{\circ} \mathrm{C}$ and vigorous stirring under the similar reaction conditions but without the addition of glutathione and $\mathrm{HAuCl}_{4}$. We discovered that there was no psDNA breakage after the comparison between lane 1 (the original psDNA) and lane 2 (the heated psDNA), which indicated that the psDNA was highly stable under the synthetic conditions. Subsequently, we analyzed the free or unreacted psDNA of the synthesized 7S-psDNA after different purification processes $(10 \mathrm{~K}$ ultrafiltration and ethanol precipitation), and no free or unreacted psDNA was detected in the solution (Lanes 3 to 5). Finally, we completely etched the synthesized 7S-psDNA-AuNPs using to extract the functionalized 7S-psDNA. The bands of psDNA-AuNPs were completely disappeared and converted into one band (Lane 6) after treatment with TCEP, which was consistent with the band of psDNA (Lane 4). Therefore, the DNA on the psDNAAuNPs remained intact without any damaged fragments bound to the surface of AuNPs. 


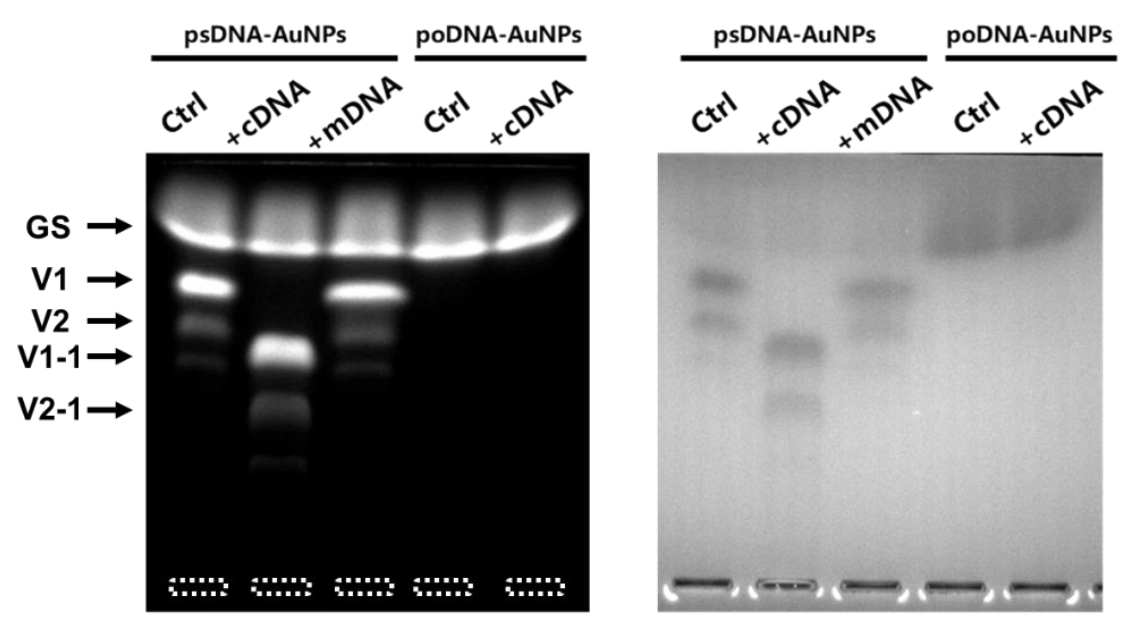

Figure S3. Characterization of the non-canonical structure or non-specific binding of psDNA-AuNPs with fully mismatched DNA (left) and the similar 7S-psDNA without phosphorothiated sites (poDNA, right). A fully mismatched DNA sequence with the similar length of cDNA (mDNA) was firstly incubated with the psDNAAuNPs. As compared with the psDNA-AuNPs incubated with cDNA, the psDNAAuNPs incubated with mDNA did not show any responsive band but remained consistent with the original psDNA-AuNPs, indicating that there was no non-canonical structure occurred between the cDNA and psDNA-AuNPs. The AuNPs synthesized with the similar 7S-psDNA without phosphorothiated sites (poDNA-AuNPs) were consistent with GS-AuNPs and did not respond to the addition of cDNA, further indicating that there was no non-specific binding between the cDNA and psDNAAuNPs. The above results also demonstrated the importance of the ps modification in the capture of gold for the formation of NIR-emitting psDNA-AuNPs. 

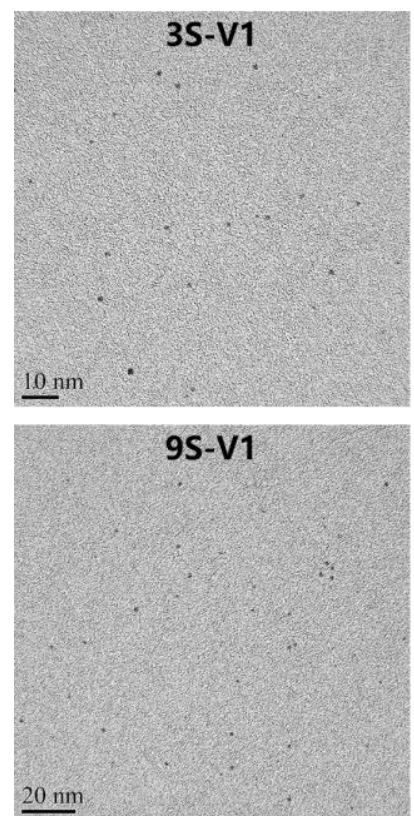
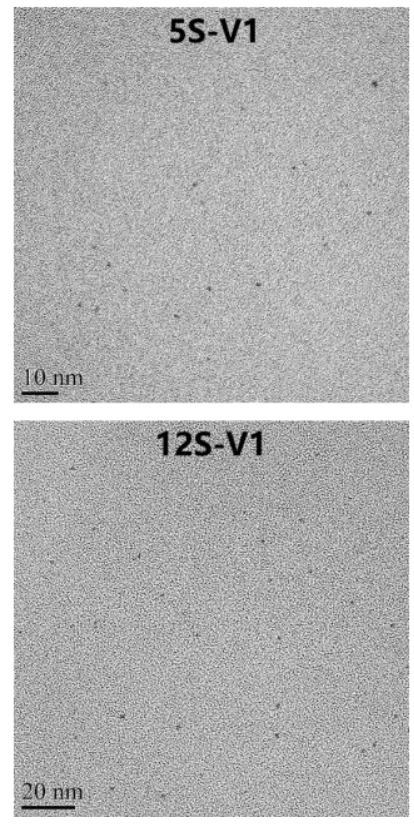

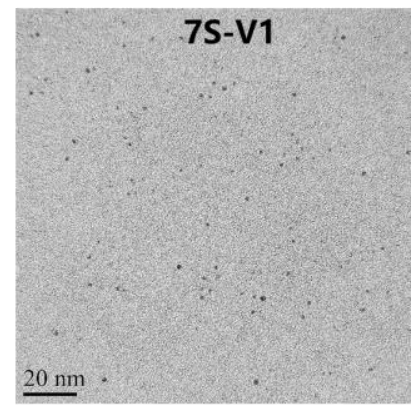

20S-V1

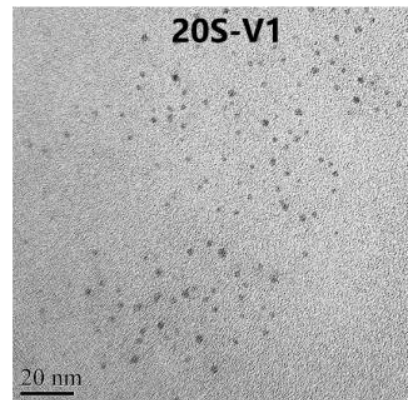

Figure S4. Typical TEM images of V1-psDNA-AuNPs synthesized using psDNA with different length of ps sites. The concept of "hinderance" is for the clustering domain of psDNA, which means the ps domain of psDNA that was depended on the number of ps sites of psDNA. If each ps site stayed linearly away from each other, the length of DNA contained ps sites could be as long as from $2.38 \mathrm{~nm}$ (7S-psDNA) to 6.8 nm (20S-psDNA), which was longer than the observed AuNP size from 1.7 (7S-psDNA) $\mathrm{nm}$ to $2.6 \mathrm{~nm}$ (20S-psDNA). Therefore, the DNA domain contained those ps sites should have a spatial configuration to make each ps site closer. Once the random ps sites reached a spatial configuration that had the enough affinities to catch the GS-Au(I) complexes, the GS-Au(I) complexes could be anchored to be reduced in the ps domain. The less ps sites could not show enough affinities to catch the GS-Au(I) complexes, and it was hard to form the luminescent psDNA-AuNPs. On the contrary, the excess ps sites could provide enough affinities, but would become more hindered for each ps site in the same psDNA to get closer to the limited size of the luminescent AuNPs, which was the intramolecular hinderance of ps domain. Besides, longer DNA also have larger size and electrostatic repulsion, which would impede two DNAs from crowding into a limited district to form a single small nanoparticle due to the intermolecular hinderance of psDNA. As evidenced from the AuNP size increasing with the increase of ps sites, the longer psDNA would form larger AuNP size and low valence. 

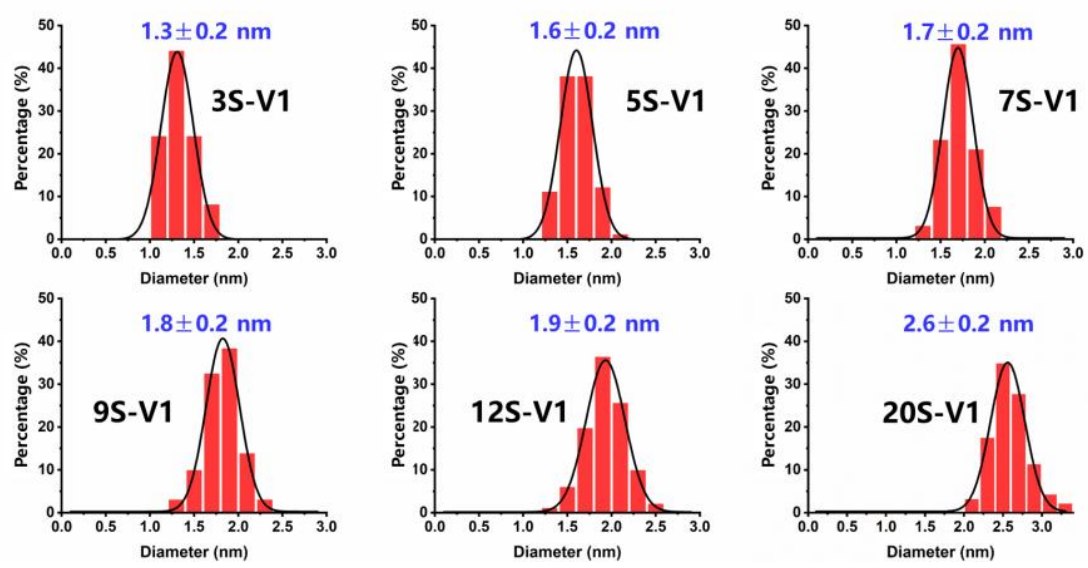

Figure S5. Size distributions of V1-psDNA-AuNPs synthesized using psDNA with different length of ps sites. The size distributions were calculated from the corresponding TEM images.

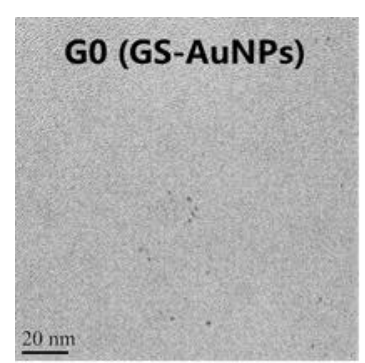

G7
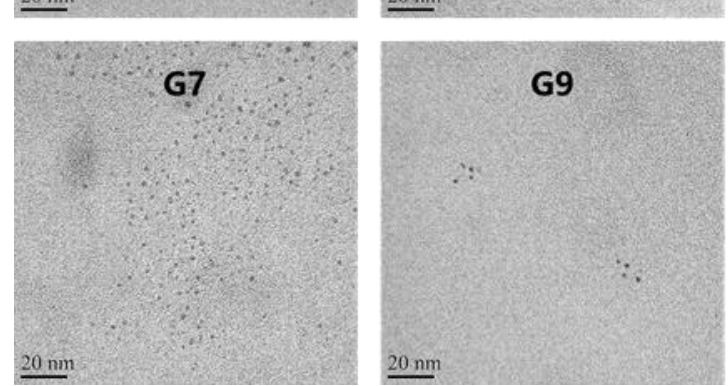

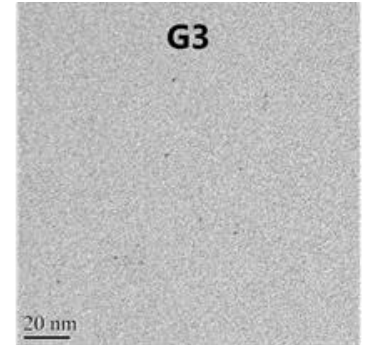

G12

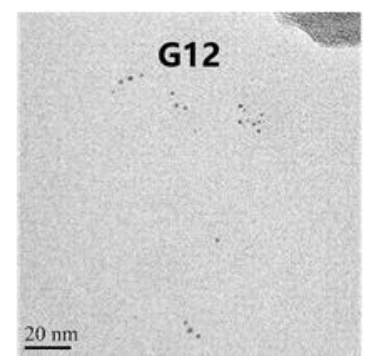

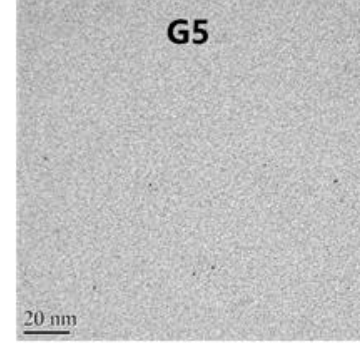

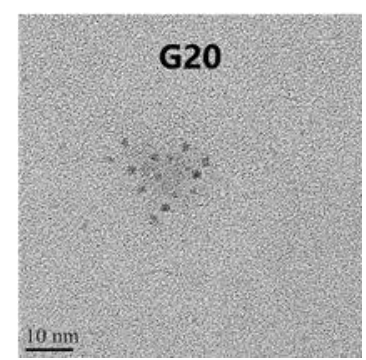

Figure S6. Typical TEM images of the GS-AuNPs fractions synthesized using psDNA with different length of ps sites. 

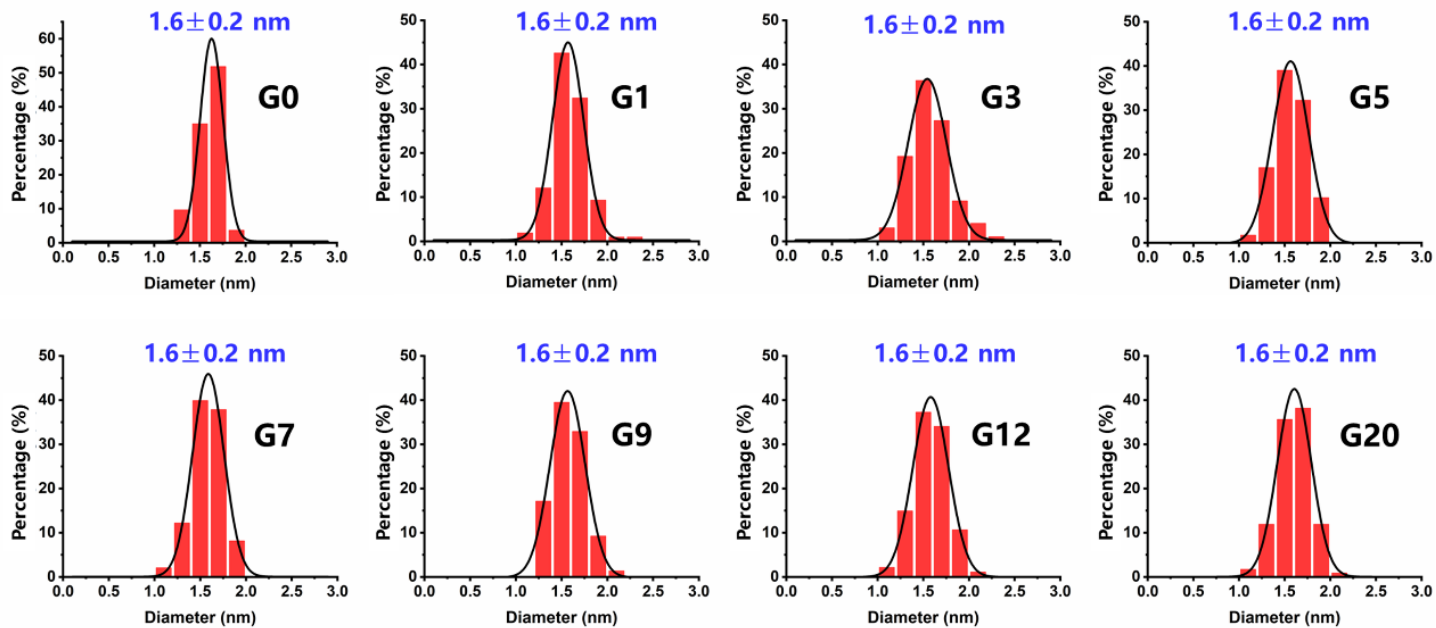

Figure S7. Size distributions of GS-AuNPs fractions synthesized using psDNA with different length of ps sites. The size distributions were calculated from the corresponding TEM images.

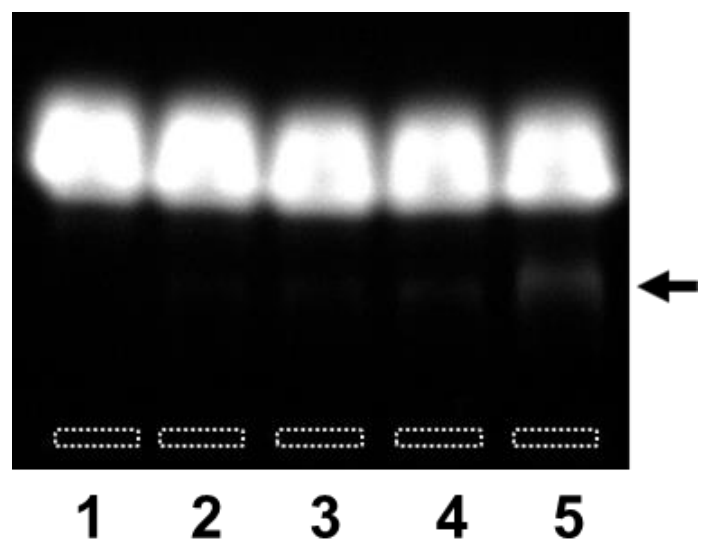

Figure S8. Determination of thiol-DNA modification of GS-AuNPs through onepot synthesis using 5'thiol-capped DNA. AGE of purified AuNPs with increase amount of cDNA from Lanes 2-5 (lane 1 as control), according to the ratios of cDNA to AuNPs (calculated by UV absorption at $350 \mathrm{~nm}$ ) of 5:1. 2:1, 1:1 and 1:20, respectively. With the addition of cDNA sequences, only $2.4 \%$ AuNPs (calculated by fluorescence gray scale statistics) was responsive to cDNA (arrow marked), and the most of AuNPs showed no response to the addition of cDNA sequences, indicating the infeasibility to synthesize DNA-modified NIR-emitting AuNPs by thiol-capped DNA. 

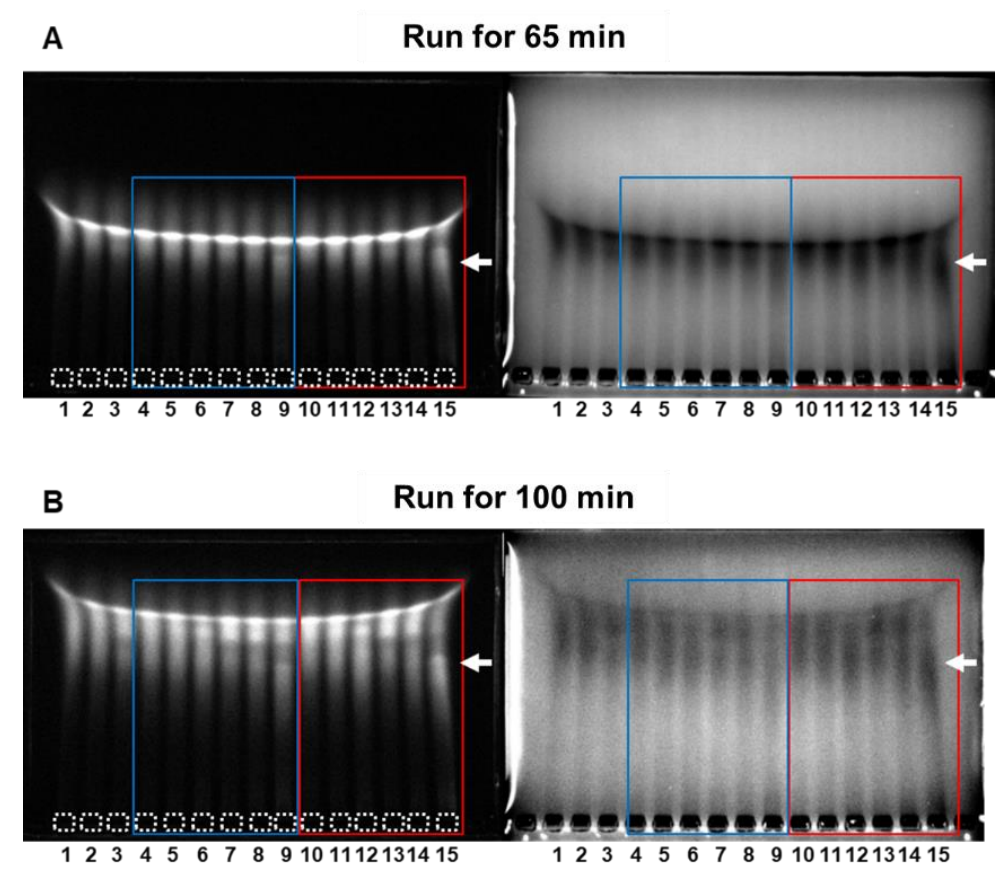

Figure S9. AGE characterization of the GS-AuNPs before and after ligand exchange with different ratios of 5'thiol/dithiol capped DNA. Lane 1: GS-AuNPs without treatment; lane 2: GS-AuNPs under ligand exchange conditions without adding 5'thiol/dithiol capped DNA; lane 3: GS-AuNPs (similar procedure as lane 2) incubated with excess cDNA; lanes 4-6: GS-AuNPs treated with 5'thiol capped DNA in the ratios of 10:1, 1:1 and 1:4 (5'thiol capped DNA/GS-AuNPs), respectively; lanes 7-9: GSAuNPs (similar as the corresponding lane 4-6) incubated with excess cDNA; lanes 1012: GS-AuNPs treated with 5'thiol capped DNA in the ratios of 10:1, 1:1 and 1:4 (5'dithiol capped DNA/ GS-AuNPs), respectively; lanes 13-15: GS-AuNPs (similar as the corresponding lane 10-12) incubated with excess cDNA. The bands of GS-AuNPs before (lane 2) and after (lanes 4-6 and lanes 10-12) the ligand exchanges with 5 'thiol/dithiol-modified DNA showed no significant differences. Therefore, it was hard to identify the DNA functionalized GS-AuNPs species. The excess cDNA was then incubated to hybridize with the DNA functionalized GS-AuNPs species to further confirm the components of DNA functionalized GS-AuNPs. After the addition of excess cDNA, only some hysteresis GS-AuNPs were observed in lanes 9 and 15, which demonstrated that it was hard to achieve controllable or distinguishable DNA functionalized luminescent AuNPs through typical thiolate ligand exchange strategy even at high concentrations of 5'thiol/dithiol-capped DNA. 


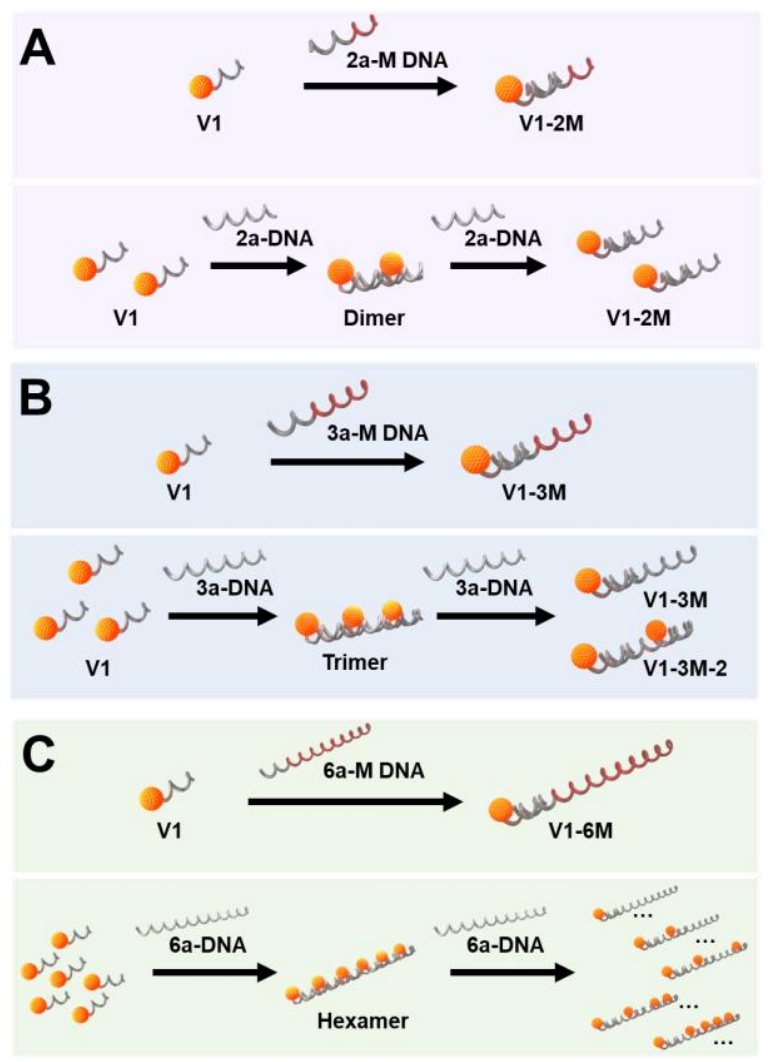

Figure S10. Schematic diagram of one-dimensional self-assemblies of psDNAAuNPs into dimer (A), trimer (B) and hexamer (C). In the self-assembly, after the addition of the repeated complementary DNA templates (2a-DNA, 3a-DNA or 6aDNA), the excess V1-psDNA-AuNPs will saturate all the DNA templates and become corresponding dimer, trimer or hexamer, but there are still unassembled V1-psDNAAuNPs in the solution. As the increase of DNA templates, the unassembled V1-psDNAAuNPs will continue to hybridize with the DNA templates, and when the stoichiometric ratios of V1-psDNA-AuNP to DNA templates reach 2:1, 3:1 and $6: 1$ for the corresponding 2a-DNA, 3a-DNA and 6a-DNA, respectively, the V1-psDNA-AuNPs are fully hybridized and become corresponding dimer, trimer and hexamer. The disassembly process occurs with the continued increase of the DNA templates. To better indicate this self-assembly and disassembly transformation process, we design three DNA sequences 2a-M DNA, 3a-M DNA and 6a-M DNA, which have identical DNA base length with the corresponding repeated complementary DNA templates but only have one complementary binding domain to the V1-psDNA-AuNPs. The above transformations of the assembled nanostructures were clearly observed in the AGE (Figure 4A-C), which further confirmed the well-controlled fabrication process of the assembled nanostructures using psDNA-AuNPs bearing strict DNA valence as building blocks. 


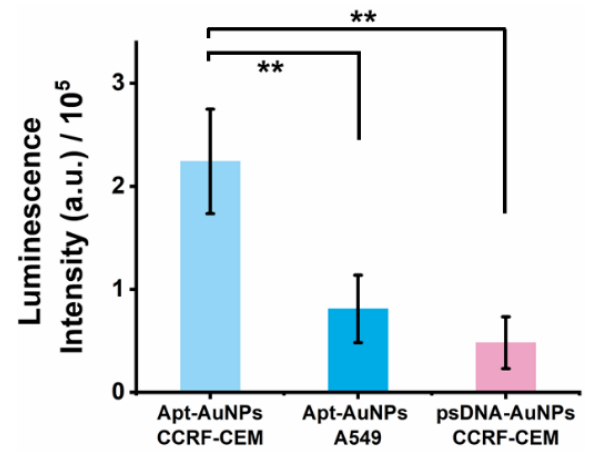

Figure S11. Fluorescence statistics of the corresponding cells. Statistical significance was assessed by a two-tailed t-test $\left(n=11\right.$ independent cells). ${ }^{* *} \mathrm{P}<0.01$.
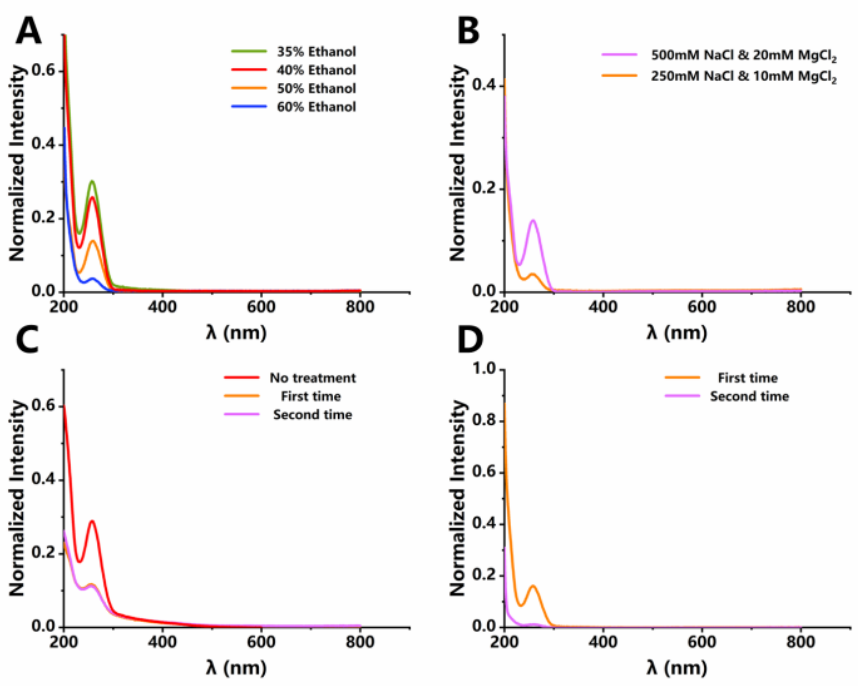

Figure S12. UV-Vis characterization of free psDNA of the psDNA-AuNPs. (A) UVVis spectra of supernatant of psDNA-AuNPs after one time of centrifugation by using different concentrations of ethanol solutions with $500 \mathrm{mM} \mathrm{NaCl}$ and $20 \mathrm{mM} \mathrm{MgCl}_{2}$. Large amount of unreacted DNA was retained in the supernatant. Since psDNA-AuNPs were precipitated in this condition, $35 \%$ ethanol solutions had the best effect on remove unreacted DNA from psDNA-AuNPs. (B) UV-Vis spectra of supernatant of psDNAAuNPs after one time of centrifugation by using $35 \%$ ethanol solutions with different concentrations of salt. Higher concentration of salt could help remove unreacted DNA better. (C) UV-Vis spectra of psDNA-AuNPs without treatment and the precipitated psDNA-AuNPs (dissolved in $1 \times$ TE buffer) after 1 or 2 times of centrifugation. Significant decrease of the absorption at $260 \mathrm{~nm}$ after 1 time of centrifugation and the little difference between 1 or 2 times of centrifugation indicated the high efficacy of this method to remove excess DNA. (D) UV-Vis spectra of supernatant after 1 or 2 times of centrifugation. There were little DNA remained in the supernatant in the second times of centrifugation, indicating most of the unreacted DNA were removed. 

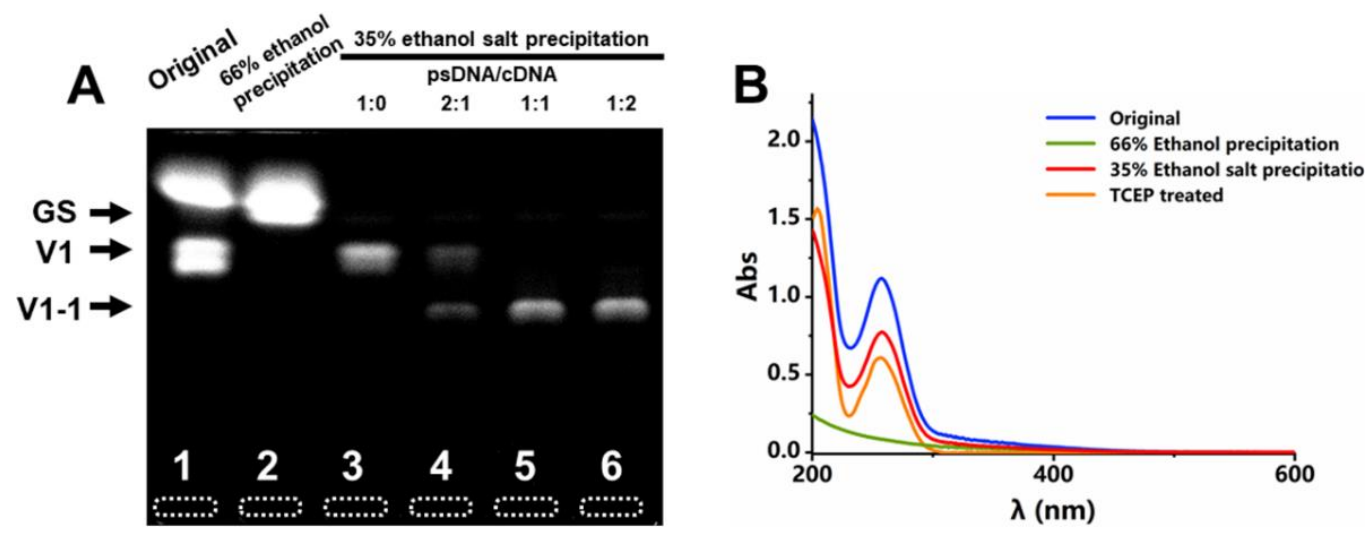

Figure S13. Characterization of the purification of 20S-psDNA-AuNPs. The as synthesized 20S-psDNA-AuNPs was firstly ultrafiltrated (10 K MWCO) to remove the excess $\mathrm{HAuCl}_{4}$ and GSH (Lane 1). The resulting 20S-psDNA-AuNPs were then mixed with $66 \%$ ethanol $(v / v)$ and centrifugated at $21000 \mathrm{~g}$ at $4{ }^{\circ} \mathrm{C}$ for $5 \mathrm{~min}$, and the precipitates were redissolve in $1 \times \mathrm{TE}$ (Lane 2). The supernatant was subsequently adjusted to $35 \%$ ethanol $(v / v)$ solution containing $500 \mathrm{mM} \mathrm{NaCl}$ and $20 \mathrm{mM} \mathrm{MgCl}_{2}$, which made the supernatant precipitated once again, followed by centrifuging at 21000 $\mathrm{g}$ at $4^{\circ} \mathrm{C}$ for $5 \mathrm{~min}$. The precipitates were redissolved in $1 \times \mathrm{TE}$ (Lane 3), and were treated with TCEP to etch all the AuNPs for the further quantification of psDNA by UV-Vis. By adding stoichiometric ratios of psDNA to cDNA (1:0, 2:1, 1:1 and 1:2 corresponding to lane 3 , lane 4 , lane 5 , and lane 6 ). The results indicated that the V1psDNA-AuNPs could be facilely purified through an ethanol precipitation purification method without gel separation, and retained the DNA bioreactivity with no free psDNA in the solution. 


\section{References}

(1) Lai, X.; Luo, F.; Wang, Y.; Su, X.; Liu, J., Coordination-Induced Decomposition of Luminescent Gold Nanoparticles: Sensitive Detection of $\mathrm{H}_{2} \mathrm{O}_{2}$ and Glucose. Anal. Bioanal. Chem. 2017, 409 (6), 1635-1641.

(2) Liu, J.; Yu, M.; Zhou, C.; Yang, S.; Ning, X.; Zheng, J., Passive Tumor Targeting of RenalClearable Luminescent Gold Nanoparticles: Long Tumor Retention and Fast Normal Tissue Clearance. J. Am. Chem. Soc. 2013, 135 (13), 4978-4981.

(3) Liu, J.; Duchesne, P. N.; Yu, M.; Jiang, X.; Ning, X.; Vinluan Iii, R. D.; Zhang, P.; Zheng, J., Luminescent Gold Nanoparticles with Size-Independent Emission. Angew. Chem., Int. Ed. 2016, 55 (31), 8894-8898.

(4) Ma, N.; Sargent, E. H.; Kelley, S. O., One-Step DNA-Programmed Growth of Luminescent and Biofunctionalized Nanocrystals. Nat. Nanotechnol. 2009, 4 (2), 121-125.

(5) Tikhomirov, G.; Hoogland, S.; Lee, P.; Fischer, A.; Sargent, E. H.; Kelley, S. O., DNABased Programming of Quantum Dot Valency, Self-Assembly and Luminescence. Nat.

Nanotechnol. 2011, 6 (8), 485.

(6) Li, Y.; Zheng, Y.; Gong, M.; Deng, Z., Pt Nanoparticles Decorated with a Discrete Number of DNA Molecules for Programmable Assembly of Au-Pt Bimetallic Superstructures. Chem. Commun. 2012, 48 (31), 3727-3729.

(7) Zheng, Y.; Li, Y.; Deng, Z., Silver Nanoparticle-DNA Bionanoconjugates Bearing a Discrete Number of DNA Ligands. Chem. Commun. 2012, 48 (49), 6160-6162. 